\title{
Effects of Statin on Small Dense Low-Density Lipoprotein Cholesterol and Remnant-Like Particle Cholesterol in Heterozygous Familial Hypercholesterolemia
}

\author{
Tsuyoshi Nozue ${ }^{1}$, Ichiro Michishita ${ }^{1}$, Yasuki Ito ${ }^{2}$, and Tsutomu Hirano ${ }^{3}$ \\ ${ }^{1}$ Division of Cardiology, Department of Internal Medicine, Yokohama Sakae Kyosai Hospital, Federation of National Public Service \\ Personnel Mutual Associations, Yokohama, Japan. \\ ${ }^{2}$ Research and Development Department, Denka Seiken Co. Ltd., Tokyo, Japan. \\ ${ }^{3}$ First Department of Internal Medicine, Showa University School of Medicine, Tokyo, Japan.
}

\begin{abstract}
Aim: The effects of statin on small dense low-density lipoprotein cholesterol (sd-LDL-C) and remnant-like particle cholesterol (RLP-C) levels in heterozygous familial hypercholesterolemia (FH) have not been examined. This study aimed to clarify the effects of statin on sd-LDL-C and RLP-C levels in heterozygous $\mathrm{FH}$.

Methods: Seventeen patients with heterozygous $\mathrm{FH}$ were randomly assigned to $2 \mathrm{mg}$ /day pitavastatin or $10 \mathrm{mg} /$ day atorvastatin. At baseline and 12 weeks after treatment with statin, we measured sd-LDL-C and RLP-C levels.

Results: Sd-LDL-C levels significantly decreased from $43 \pm 24$ to $16 \pm 10 \mathrm{mg} / \mathrm{dL}(-63 \%, p=0.001)$ in the pitavastatin group, and from $44 \pm 17$ to $19 \pm 10 \mathrm{mg} / \mathrm{dL}(-55 \%, p<0.001)$ in the atorvastatin group. RLP-C levels decreased from $8.4 \pm 2.8$ to $6.6 \pm 2.7 \mathrm{mg} / \mathrm{dL}(-16 \%, p=0.156)$ in the pitavastatin group, and from $9.8 \pm 4.7$ to $5.9 \pm 5.4 \mathrm{mg} / \mathrm{dL}(-45 \%, p=0.044)$ in the atorvastatin group. There were no significant differences in percent changes of sd-LDL-C $(p=0.370)$ and RLP-C levels $(p=0.097)$ between the two groups.

Conclusions: Sd-LDL-C measured by the heparin-magnesium precipitation method and RLP-C levels in heterozygous FH were decreased by 12 weeks of statin therapy. Statin might have additional anti-atherogenic effects by reducing not only LDL-C but also sd-LDL-C and RLP-C.
\end{abstract}

J Atheroscler Thromb, 2008; 15:146-153.

Key words; Familial hypercholesterolemia, Small dense low-density lipoprotein cholesterol, Remnant-like particle cholesterol, Statin

\section{Introduction}

Familial hypercholesterolemia (FH) is a common autosomal dominant disorder caused by a mutation of the gene for the low-density lipoprotein (LDL) recep$\operatorname{tor}^{1}{ }^{1}$. FH is frequently associated with premature coronary artery disease (CAD), and the rate of death from

Address for correspondence: Tsuyoshi Nozue, Division of Cardiology, Department of Internal Medicine, Yokohama Sakae Kyosai Hospital, Federation of National Public Service Personnel Mutual Associations, 132 Katsura-cho, Sakae-ku, Yokohama 247-8581, Japan.

E-mail: nozue2493@yahoo.co.jp

Received: October 18, 2007

Accepted for publication: January 21, 2008
CAD is several times higher than the general population $^{1,2)}$; however, there is a wide variation of coronary risk among $\mathrm{FH}$ patients ${ }^{3)}$.

Increasing experimental and clinical evidence suggests that disturbed plasma triglyceride (TG) metabolism plays an important role in the development of premature atherosclerosis ${ }^{4,5)}$. Disturbances in TG metabolism are characterized by postprandial accumulation of lipoprotein remnants. A new remnant lipoprotein method based on the immunoseparation principle [remnant-like particle (RLP) cholesterol (RLP-C) assay] offers the possibility of separating lipoprotein remnant particles with the use of an immunoaffinity gel with coupled monoclonal antibodies against apoB100 and apoA-I ${ }^{6,7)}$. Increased levels of RLP-C have 
been associated with the development of $\mathrm{CAD}^{8-10)}$. FH patients with disturbances in postprandial lipoprotein metabolism also have higher risks for CAD ${ }^{11)}$.

Small dense (sd) LDL has been demonstrated to be a new risk factor for the development of CAD in Westerners as well as in Japanese ${ }^{12-14)}$. LDL particle size is usually measured by gradient gel electrophoresis using non-denaturing polyacrylamide ${ }^{15}$. Recently, Hirano et al. established a simple and rapid method for the measurement of sd-LDL-C concentrations using heparin-magnesium precipitation ${ }^{16,17)}$. This method is useful for evaluating qualitative and quantitative abnormalities in LDL, and may be applicable to a routine clinical examination ${ }^{18)}$.

Pitavastatin (Kowa Pharmaceutical Co. Ltd., Tokyo, Japan) is new 3-hydroxy-3-methylglutaryl coenzyme A (HMG-CoA) reductase inhibitor (statin) developed in Japan. Total cholesterol (TC) and LDL-C levels in heterozygous $\mathrm{FH}$ decreased by $31 \%$ and $40 \%$ with 2 $\mathrm{mg} /$ day pitavastatin, respectively ${ }^{19)}$. It remains unclear, however, whether statin could reduce sd-LDL-C by mechanisms similar to LDL-C. Sd-LDL-C and RLP-C levels and the response to statin therapy could be relevant for understanding the heterogeneity in coronary risk among FH patients. The goal of this study was to assess the effects of statin therapy on sd-LDL-C and RLP-C levels in heterozygous FH. Furthermore, the comparative effects of two statins (pitavastatin and atorvastatin), both of which appeared to decrease TG levels ${ }^{19-21)}$, were evaluated.

\section{Materials and Methods}

\section{Subjects}

Seventeen patients with heterozygous $\mathrm{FH}$ (male/ female $=3 / 14$, mean age $=61 \pm 11$ years) were studied. $\mathrm{FH}$ was diagnosed according to the following two criteria: (1) primary hypercholesterolemic patients (TC level above $230 \mathrm{mg} / \mathrm{dL}$ in any age group) with tendon xanthomas, or (2) primary hypercholesterolemic patients with and without tendon xanthomas in a first-degree relative of familial hypercholesterolemic patients ${ }^{22}$. After a washout period of 8 weeks, patients were randomly assigned to $2 \mathrm{mg} /$ day pitavastatin or $10 \mathrm{mg} /$ day atorvastatin. At baseline and 12 weeks after treatment with statin, we measured sd-LDL-C and RLP-C levels. Written informed consent was obtained from all patients.

\section{Laboratory Determination}

Blood samples were obtained after an overnight fast. Serum TC and TG levels were measured by standard enzymatic methods. Serum LDL-C and high-den- sity lipoprotein cholesterol (HDL-C) levels were measured by direct homogeneous assay using detergents (LDL-EX and HDL-EX, Denka Seiken Co. Ltd., Tokyo). Sd-LDL-C level was measured using a commercially available test kit (sd-LDL-C "Seiken", Denka Seiken Co. Ltd.). The details and validation of this method have been described elsewhere ${ }^{16,17)}$. Serum RLP-C level was measured using an immune-separation technique (Otsuka Pharmaceuticals Co., Ltd., To$\left.\mathrm{kyo}^{7}\right)^{7}$. Serum lipoprotein (a) $[\mathrm{Lp}(\mathrm{a})]$ levels were assayed by a commercially available enzyme-linked immunosorbent assay (Daiichi Pure Chemicals Co., Ltd., Tokyo ${ }^{23)}$. Large buoyant (lb)-LDL-C was calculated by subtracting sd-LDL-C from LDL-C.

\section{Statistical Analysis}

Statistical analyses were performed using Statview 5.0 software (SAS Institute, Cary, NC, USA). All values are expressed as the mean $\pm S D$. Differences between the means were compared with the unpaired $t$-test. The significance of any differences in proportions was tested with Chi-square analysis. One-way analysis of variance (ANOVA) was used to compare mean changes of lipid parameters, and multiple comparisons were made by Bonferroni's test. Significant difference was defined as $p<0.05$.

\section{Results}

The baseline characteristics of the patients are shown in Table 1. Three patients were male, and 14 patients were female. The mean age was $61 \pm 11$ years. Mean TC, LDL-C, sd-LDL-C, and RLP-C levels at baseline were $303 \pm 45 \mathrm{mg} / \mathrm{dL}, 218 \pm 47 \mathrm{mg} / \mathrm{dL}, 44$ $\pm 20 \mathrm{mg} / \mathrm{dL}$, and $9.2 \pm 3.9 \mathrm{mg} / \mathrm{dL}$, respectively. There were no significant differences in age, gender, body mass index, TC, LDL-C, HDL-C, TG, sd-LDL-C, and RLP-C levels between the two groups.

Mean LDL-C and apo B levels 12 weeks after treatment with pitavastatin decreased significantly from $201 \pm 27$ to $111 \pm 11 \mathrm{mg} / \mathrm{dL}(-45 \%, p<0.0001)$ and from $160 \pm 9$ to $101 \pm 11 \mathrm{mg} / \mathrm{dL}(-37 \%, p<0.0001)$. Mean LDL-C and apo B levels 12 weeks after treatment with atorvastatin decreased significantly from $234 \pm 57$ to $142 \pm 52 \mathrm{mg} / \mathrm{dL}(-40 \%, p=0.001)$ and from $170 \pm 38$ to $114 \pm 32 \mathrm{mg} / \mathrm{dL}(-34 \%, p<0.001)$. Serum high-sensitivity C-reactive protein (hs-CRP) levels tended to be decreased by statin therapy, but were not statistically significant (Table 2).

Sd-LDL-C levels 12 weeks after treatment with statin significantly decreased from $43 \pm 24$ to $16 \pm 10$ $\mathrm{mg} / \mathrm{dL}(-63 \%, p=0.001)$ in the pitavastatin group and from $44 \pm 17$ to $19 \pm 10 \mathrm{mg} / \mathrm{dL}(-55 \%, p<0.001)$ 
Table 1. Baseline characteristics

\begin{tabular}{|c|c|c|c|c|}
\hline & $\begin{array}{l}\text { All patients } \\
(n=17)\end{array}$ & $\begin{array}{c}\text { Pitavastatin } \\
\quad(n=8)\end{array}$ & $\begin{array}{l}\text { Atorvastatin } \\
\quad(n=9)\end{array}$ & $p$ value \\
\hline Age (years) & $61 \pm 11$ & $62 \pm 6$ & $60 \pm 15$ & 0.758 \\
\hline Gender (male/female) & $3 / 14$ & $1 / 7$ & $2 / 7$ & 0.600 \\
\hline Height $(\mathrm{cm})$ & $157.3 \pm 9.4$ & $159.0 \pm 7.6$ & $155.8 \pm 11.1$ & 0.500 \\
\hline Body weight (kg) & $56.5 \pm 9.7$ & $58.0 \pm 7.0$ & $55.2 \pm 11.9$ & 0.566 \\
\hline BMI $\left(\mathrm{kg} / \mathrm{m}^{2}\right)$ & $22.7 \pm 2.5$ & $22.9 \pm 1.7$ & $22.6 \pm 3.2$ & 0.790 \\
\hline $\mathrm{TC}(\mathrm{mg} / \mathrm{dL})$ & $303 \pm 45$ & $286 \pm 32$ & $318 \pm 51$ & 0.146 \\
\hline LDL-C (mg/dL) & $218 \pm 47$ & $201 \pm 27$ & $234 \pm 57$ & 0.167 \\
\hline HDL-C (mg/dL) & $60 \pm 13$ & $58 \pm 15$ & $62 \pm 11$ & 0.514 \\
\hline $\mathrm{TG}(\mathrm{mg} / \mathrm{dL})$ & $152 \pm 60$ & $165 \pm 64$ & $141 \pm 58$ & 0.443 \\
\hline RLP-C (mg/dL) & $9.2 \pm 3.9$ & $8.4 \pm 2.8$ & $9.8 \pm 4.7$ & 0.477 \\
\hline $\mathrm{Lp}(\mathrm{a})(\mathrm{mg} / \mathrm{dL})$ & $33 \pm 27$ & $27 \pm 23$ & $37 \pm 31$ & 0.462 \\
\hline sd-LDL-C (mg/dL) & $44 \pm 20$ & $43 \pm 24$ & $44 \pm 17$ & 0.859 \\
\hline lb-LDL-C (mg/dL) & $175 \pm 39$ & $159 \pm 28$ & $189 \pm 43$ & 0.113 \\
\hline Smoking (\%) & $4(24 \%)$ & $2(25 \%)$ & $2(22 \%)$ & 0.893 \\
\hline History of CAD (\%) & $4(24 \%)$ & $2(25 \%)$ & $2(22 \%)$ & 0.893 \\
\hline History of CVA (\%) & $1(6 \%)$ & $0(0 \%)$ & $1(11 \%)$ & 0.331 \\
\hline $\mathrm{DM}(\%)$ & $2(12 \%)$ & $0(0 \%)$ & $2(22 \%)$ & 0.156 \\
\hline Hypertension (\%) & $4(24 \%)$ & $2(25 \%)$ & $2(22 \%)$ & 0.893 \\
\hline
\end{tabular}

All values are expressed as the mean \pm SD or number (\%).

BMI, body mass index; TC, total cholesterol; LDL-C, low-density lipoprotein cholesterol; HDL-C, high-density lipoprotein cholesterol; TG, triglyceride; RLP-C, remnant-like particle cholesterol; Lp(a), lipoprotein (a); sd-LDL-C, small dense low-density lipoprotein cholesterol; lb-LDL-C, large buoyant low-density lipoprotein cholesterol; CAD, coronary artery disease; CVA, cerebrovascular accident; DM, diabetes mellitus.

in the atorvastatin group (Table 2). Fig. 1 shows serial changes in sd-LDL-C levels during statin therapy. There was variability in the baseline sd-LDL-C levels. The highest and lowest levels of sd-LDL-C were 95 $\mathrm{mg} / \mathrm{dL}$ and $24 \mathrm{mg} / \mathrm{dL}$. Sd-LDL-C levels decreased significantly 4 weeks after treatment with statin and remained depressed to 12 weeks in both groups.

Fig. 2 shows serial changes in RLP-C levels during statin therapy. RLP-C levels decreased significantly 4 weeks after treatment with statin and tended to be depressed to 12 weeks in both groups.

Percent changes in LDL-C, apo B, sd-LDL-C, lbLDL-C, and RLP-C levels after 12 weeks of statin therapy were $-42 \%,-35 \%,-59 \%,-38 \%$, and $-31 \%$, respectively (Fig. 3). There were no significant differences in the reduction of sd-LDL-C $(p=0.370)$ and RLP-C levels between the two groups $(p=0.097)$. Percent changes of sd-LDL-C levels were higher than percent changes of $\mathrm{lb}-\mathrm{LDL}-\mathrm{C}$ levels in both groups. In simple linear regression analysis, mean changes of sdLDL-C were positively correlated with mean changes of TG $(r=0.828 . p=0.011)$ in the pitavastatin group, but not in the atorvastatin group $(r=0.146, p=0.708)$ (Table 3). Mean changes of RLP-C levels were positively correlated with mean changes of TG in both groups $(r=0.823, p=0.012$ in the pitavastatin group and $r=0.751, p=0.020$ in the atorvastatin group) (Table 4).

\section{Discussion}

This study is the first report to examine the effects of statin on sd-LDL-C and RLP-C levels in heterozygous $\mathrm{FH}$. These results suggested that sd-LDL-C and RLP-C levels in heterozygous FH were decreased by statin therapy.

Sd-LDL-C levels have been positively correlated with both TG and LDL-C levels and inversely correlated with HDL-C levels ${ }^{18}$; however, the most powerful determinant of LDL size may be TG levels ${ }^{24}$. It has been demonstrated that cholesteryl ester transfer protein (CETP) is also an important determinant of LDL particle size ${ }^{25,26)}$. In addition, lipoprotein lipase (LPL) activity and hepatic lipase activity have been shown to contribute to the formation of sd-LDL particles and could well represent significant determinants of LDL-peak particle diameter (LDL-PPD) in $\mathrm{FH}^{27)}$. In the previous report, LDL-PPD in $\mathrm{FH}$ was significantly smaller than in controls. FH subjects had $11 \%$ higher CETP mass and there was an inverse rela- 
Table 2. Effects of statins on lipid and apolipoprotein levels

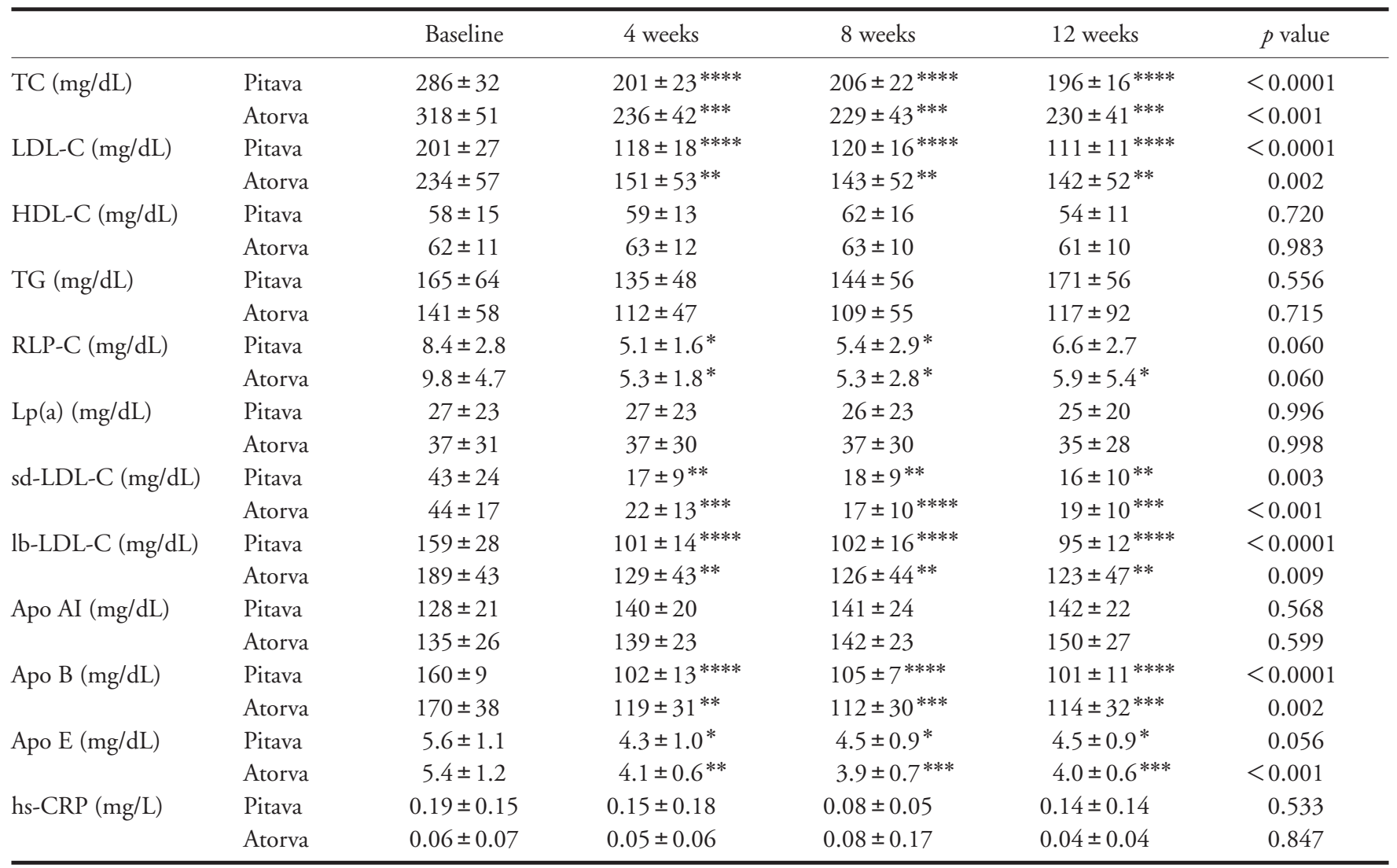

All values are expressed as the mean \pm SD.

Pitava, pitavastatin; Atorva, atorvastatin; TC, total cholesterol; LDL-C, low-density lipoprotein cholesterol; HDL-C, high-density lipoprotein cholesterol; TG, triglyceride; RLP-C, remnant-like particle cholesterol; Lp(a), lipoprotein (a); sd-LDL-C, small dense low-density lipoprotein cholesterol; lb-LDL-C, large buoyant low-density lipoprotein cholesterol; Apo, apolipoprotein; hs-CRP, high-sensitivity C-reactive protein.

One-way analysis of variance (ANOVA) was performed for each variable.

Multiple comparisons were made by Bonferroni’s test.

${ }^{*} p<0.05,{ }^{* *} p<0.01,{ }^{* * *} p<0.001,{ }^{* * * *} p<0.0001$ vs. baseline.

A

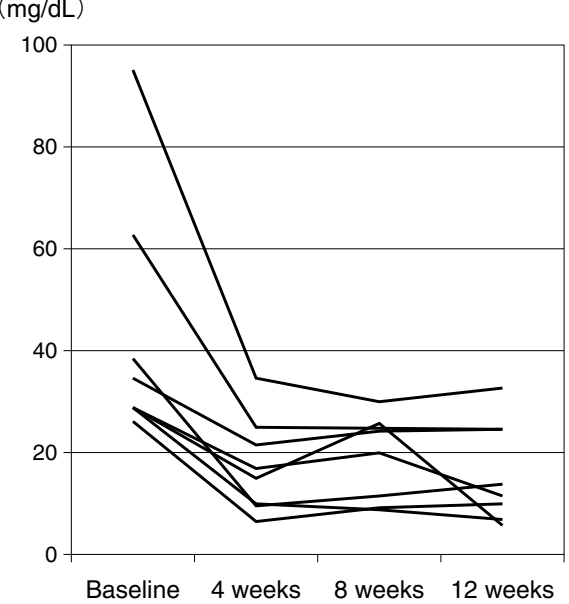

B

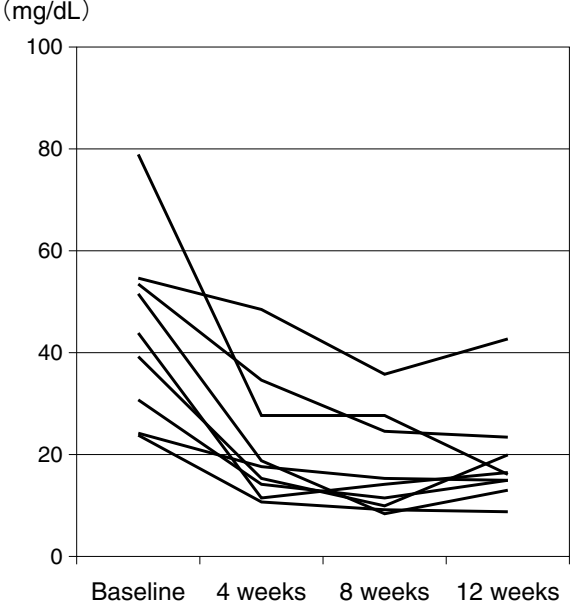

Fig. 1. Serial changes in small dense low-density lipoprotein cholesterol levels during statin therapy. (A): Pitavastatin group, (B): Atorvastatin group. 
A

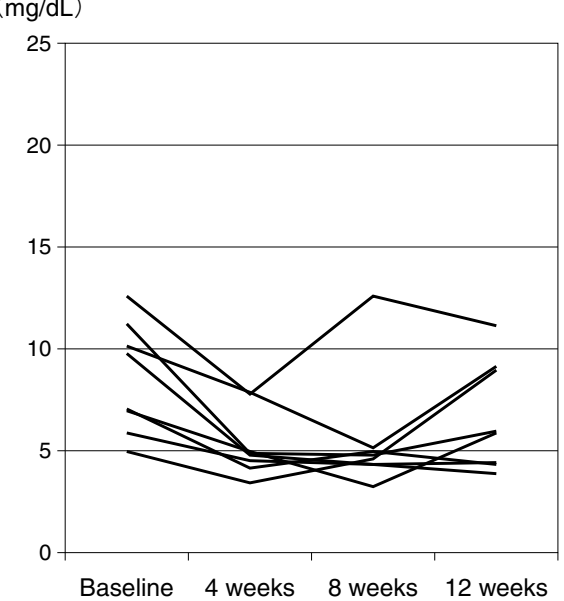

B

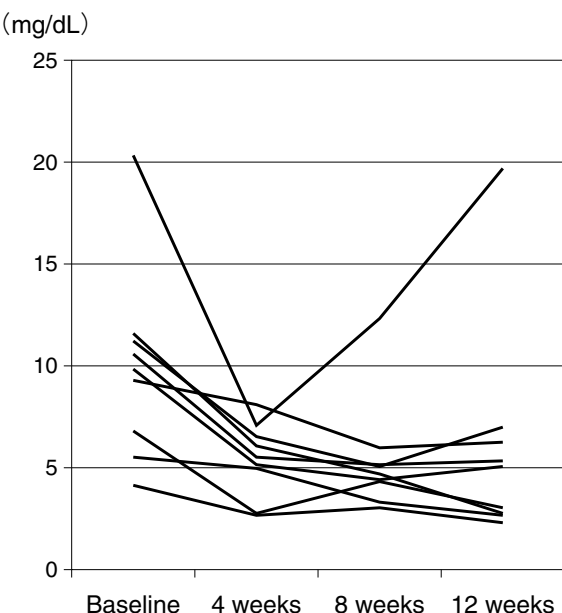

Fig. 2. Serial changes in remnant-like particle cholesterol levels during statin therapy.

(A): Pitavastatin group, (B): Atorvastatin group.

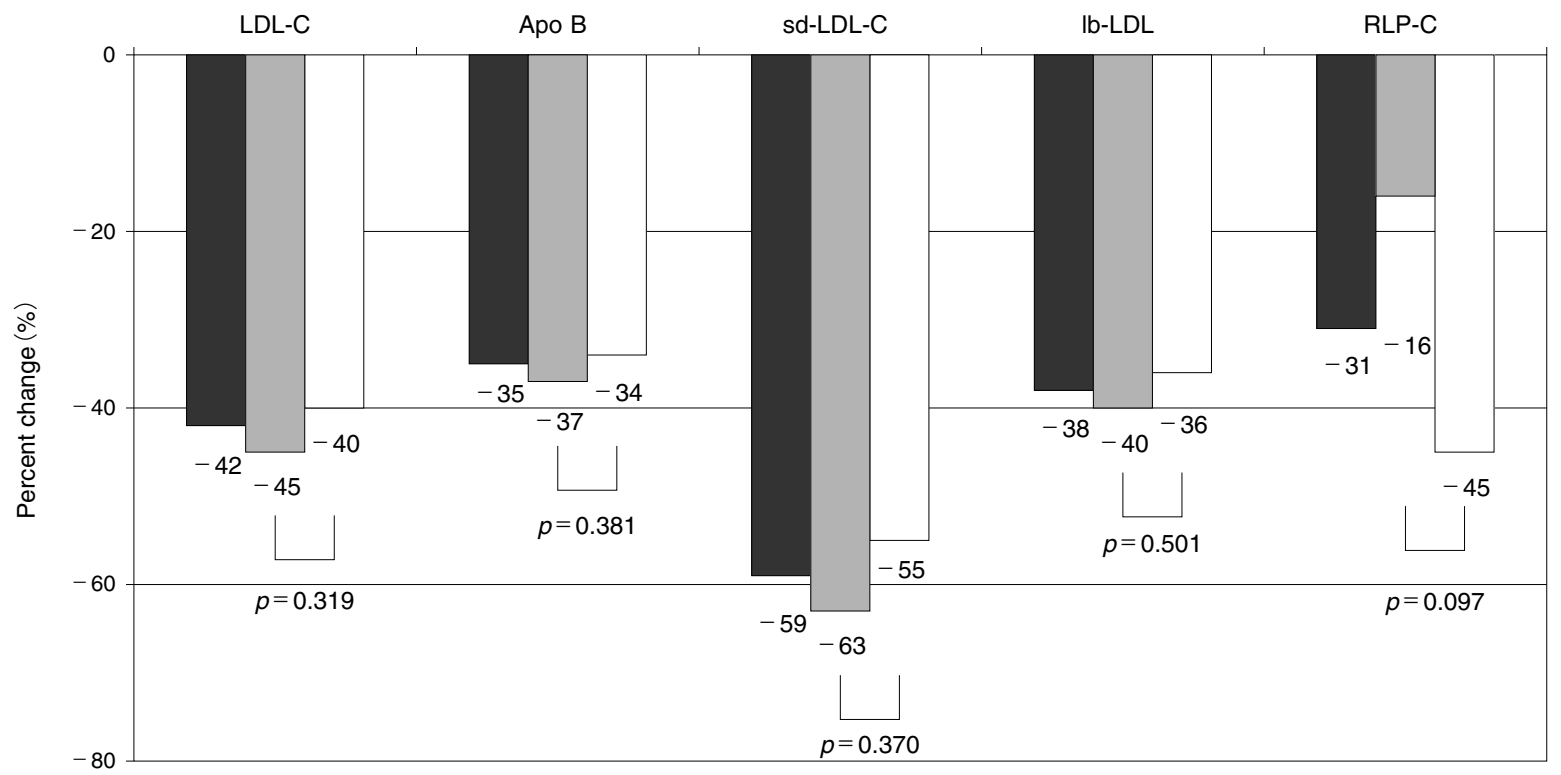

Fig. 3. Percent changes in LDL-C, apo B, sd-LDL-C, lb-LDL-C, and RLP-C levels after 12 weeks of statin therapy.

$\square$ : All patients, $\square$ : Pitavastatin group, $\square$ : Atorvastatin group.

LDL-C, low-density lipoprotein cholesterol; Apo, apolipoprotein; sd-LDL-C, small dense low-density lipoprotein cholesterol; lb-LDL-C, large buoyant low-density lipoprotein cholesterol; RLP-C, remnant-like particle cholesterol.

tionship between LDL-PPD and CETP mass. TG levels and CETP mass concentrations, as well as gender were independent predictors of LDL-PPD and represented nearly $27 \%$ of its variance in $\mathrm{FH}^{28)}$. These findings indicate that a large proportion of the variability in LDL-PPD remained unexplained by these parameters. Hogue et al. reported that the genotype of the LDL receptor was associated with LDL particle size heterogeneity in FH. They showed that the LDL receptor status accounted for $5.7 \%$ of the variance in the LDL-PPD ${ }^{29)}$. In the present study, the highest and lowest levels of sd-LDL-C at baseline were $95 \mathrm{mg} / \mathrm{dL}$ and $24 \mathrm{mg} / \mathrm{dL}$, revealing the variability of sd-LDL-C levels in FH. We previously reported that sd-LDL-C determined by this method was a useful marker of metabolic syndrome (MetS) in patients with $\mathrm{CAD}^{30)}$. In this report, mean levels of sd-LDL-C with MetS were $26.4 \pm 2.6 \mathrm{mg} / \mathrm{dL}$. Tokuno et al. reported that 
Table 3. Simple linear regression analysis between mean change of small dense low-density lipoprotein cholesterol levels and various parameters

\begin{tabular}{|c|c|c|c|c|}
\hline \multirow{2}{*}{ Variables } & \multicolumn{2}{|c|}{ Pitavastatin } & \multicolumn{2}{|c|}{ Atorvastatin } \\
\hline & $r$ & $p$ value & $r$ & $p$ value \\
\hline$\Delta \mathrm{TC}(\mathrm{mg} / \mathrm{dL})$ & 0.125 & 0.768 & 0.333 & 0.381 \\
\hline$\Delta \mathrm{LDL}-\mathrm{C}(\mathrm{mg} / \mathrm{dL})$ & 0.446 & 0.268 & 0.263 & 0.494 \\
\hline$\Delta \mathrm{HDL}-\mathrm{C}(\mathrm{mg} / \mathrm{dL})$ & 0.037 & 0.931 & 0.065 & 0.868 \\
\hline$\Delta \mathrm{TG}(\mathrm{mg} / \mathrm{dL})$ & 0.828 & 0.011 & 0.146 & 0.708 \\
\hline$\Delta$ Apo AI (mg/dL) & 0.115 & 0.787 & 0.127 & 0.746 \\
\hline$\Delta$ Apo B (mg/dL) & 0.365 & 0.374 & 0.165 & 0.671 \\
\hline$\Delta$ Apo E (mg/dL) & 0.382 & 0.351 & 0.425 & 0.255 \\
\hline$\Delta \mathrm{lb}-\mathrm{LDL}-\mathrm{C}(\mathrm{mg} / \mathrm{dL})$ & 0.300 & 0.471 & 0.627 & 0.071 \\
\hline$\Delta \mathrm{RLP}-\mathrm{C}(\mathrm{mg} / \mathrm{dL})$ & 0.804 & 0.016 & 0.123 & 0.752 \\
\hline
\end{tabular}

TC, total cholesterol; LDL-C, low-density lipoprotein cholesterol; HDL-C, high-density lipoprotein cholesterol; TG, triglyceride; Apo, apolipoprotein; lb-LDL-C, large buoyant low-density lipoprotein cholesterol; RLP-C, remnant-like particle cholesterol.

sd-LDL-C levels determined by this method were 37 $\pm 20 \mathrm{mg} / \mathrm{dL}$ in hypercholesterolemic subjects with type 2 diabetes and $45 \pm 21 \mathrm{mg} / \mathrm{dL}$ in hypertriglyceridemic subjects with type 2 diabetes ${ }^{31}$. Compared with these data, mean levels of sd-LDL-C $(44 \pm 20 \mathrm{mg} / \mathrm{dL})$ in heterozygous $\mathrm{FH}$ were equal to or more than in subjects with MetS or type 2 diabetes.

As several statins have been known to decrease sd-LDL levels ${ }^{32)}$ or increase LDL particle size ${ }^{33-35)}$, our data also indicate the effect of statin on lowering sdLDL-C levels in heterozygous FH. Percent changes of sd-LDL-C levels were $63 \%$ in the pitavastatin group and $55 \%$ in the atorvastatin group. Thus, the effect of statin on lowering sd-LDL-C levels was similar in both $2 \mathrm{mg} /$ day of pitavastatin and $10 \mathrm{mg} /$ day of atorvastatin.

In the present study, RLP-C levels in FH were decreased by statin therapy. Furthermore, mean changes of RLP-C levels were positively correlated with mean changes of TG in both groups. This observation was reported previously ${ }^{36,37)}$; however, not all statins could reduce RLP-C levels. Stein et al. reported that RLP-C levels were reduced by simvastatin $20 \mathrm{mg}$ and by atorvastatin $10 \mathrm{mg}$ but not by pravastatin $40 \mathrm{mg}$ in patients with combined dyslipidemia ${ }^{38)}$. A recent study indicated that the LDL receptor may regulate the rate of entrance of very low-density lipoprotein (VLDL) into the circulation ${ }^{39)}$, indicating that reduced RLP-C levels might be mainly due to decreasing hepatic VLDL synthesis in both statins.

On the other hand, mean changes of sd-LDL-C levels were positively correlated with mean changes of TG in the pitavastatin group, but not in the atorva-
Table 4. Simple linear regression analysis between mean change of remnant-like particle cholesterol levels and various parameters

\begin{tabular}{|c|c|c|c|c|}
\hline \multirow{2}{*}{ Variables } & \multicolumn{2}{|c|}{ Pitavastatin } & \multicolumn{2}{|c|}{ Atorvastatin } \\
\hline & $r$ & $p$ value & $r$ & $p$ value \\
\hline$\Delta \mathrm{TC}(\mathrm{mg} / \mathrm{dL})$ & 0.035 & 0.934 & 0.430 & 0.248 \\
\hline$\Delta \mathrm{LDL}-\mathrm{C}(\mathrm{mg} / \mathrm{dL})$ & 0.294 & 0.480 & 0.410 & 0.273 \\
\hline$\Delta \mathrm{HDL}-\mathrm{C}(\mathrm{mg} / \mathrm{dL})$ & 0.151 & 0.721 & 0.101 & 0.797 \\
\hline$\Delta \mathrm{TG}(\mathrm{mg} / \mathrm{dL})$ & 0.823 & 0.012 & 0.751 & 0.020 \\
\hline$\Delta$ Apo AI (mg/dL) & 0.030 & 0.945 & 0.161 & 0.679 \\
\hline$\Delta$ Apo B (mg/dL) & 0.293 & 0.481 & 0.460 & 0.213 \\
\hline$\Delta$ Apo E (mg/dL) & 0.431 & 0.287 & 0.419 & 0.262 \\
\hline$\Delta \mathrm{lb}-\mathrm{LDL}-\mathrm{C}(\mathrm{mg} / \mathrm{dL})$ & 0.310 & 0.455 & 0.228 & 0.556 \\
\hline$\Delta \mathrm{sd}-\mathrm{LDL}-\mathrm{C}(\mathrm{mg} / \mathrm{dL})$ & 0.804 & 0.016 & 0.123 & 0.752 \\
\hline
\end{tabular}

TC, total cholesterol; LDL-C, low-density lipoprotein cholesterol; HDL-C, high-density lipoprotein cholesterol; TG, triglyceride; Apo, apolipoprotein; lb-LDL-C, large buoyant low-density lipoprotein cholesterol; sd-LDL-C, small dense low-density lipoprotein cholesterol.

statin group. It is possible that the mechanisms of decreased sd-LDL-C levels might be different in both statins. Furthermore, percent changes of sd-LDL-C levels were higher than percent changes of $\mathrm{lb}-\mathrm{LDL}-\mathrm{C}$ levels in both statins. Sd-LDL particles have weaker affinity to LDL receptors than $\mathrm{lb}-\mathrm{LDL}$ particles ${ }^{40)}$; however, a recent study indicated that the induction of LDL receptors by statin stimulated the uptake of all LDL particles, irrespective of their size ${ }^{31)}$. According to this report, a low dose of pitavastatin $(1 \mathrm{mg})$ decreased sd-LDL-C levels by $26 \%$ and lb-LDL-C levels by $22 \%$. We speculate that the effect of statin on lowering sd-LDL-C was not only by upregulating LDL receptors but also by decreasing hepatic VLDL synthesis and increasing LPL activities. In previous studies, statin reduced CETP levels ${ }^{41)}$ and increased LPL activity $^{21)}$. These factors may partially contribute to the reduction of sd-LDL-C levels. Lariviere et al. reported that $41.6 \%$ of the variation in the LDL-PPD response to atorvastatin was attributable to the initial LDL-PPD, apo E polymorphism, nature of the LDL receptor gene mutation, and change in TG levels ${ }^{33)}$. Thus, genetic and multiple factors associated with lipoprotein metabolism may be important determinants of statin-induced changes of LDL heterogeneity.

Sd-LDL display enhanced susceptibility to copper transition metal-induced oxidation when compared with normal-sized LDL particles ${ }^{42,43)}$, and it is recognized that oxidized LDLs are highly atherogenic ${ }^{44)}$. Thus, sd-LDL particles have been associated with the development of CAD ${ }^{12-14)}$. Furthermore, increased levels of RLP-C have been associated with the develop- 
ment of CAD ${ }^{8-10)}$. Sd-LDL-C and RLP-C might play an important role in the acceleration of atherosclerosis in heterozygous $\mathrm{FH}$. Thus, pitavastatin and atorvastatin have anti-atherogenic effects by reducing not only LDL-C levels but also sd-LDL-C and RLP-C levels.

In conclusion, sd-LDL-C measured by the heparin-magnesium precipitation method and RLP-C levels in heterozygous FH were decreased by 12 weeks of statin therapy. Statin might have additional anti-atherogenic effects by reducing not only LDL-C but also sd-LDL-C and RLP-C.

\section{Study Limitations}

There are some limitations regarding the interpretation of these results and drawing conclusions. This study included only 17 patients with heterozygous FH. The sample size allowed for only limited analysis of sd-LDL-C and RLP-C levels and response to statin therapy. Furthermore, we did not perform genetic analysis of the LDL receptor, nor were CETP concentrations and LPL activities measured.

\section{References}

1) Goldstein JL, Hobbs HH, and Brown MS: Familial hypercholesterolemia. In: The Metabolic Basis of Inherited Disease. 8th Ed, ed by Scriver CR, Beaudet AL, Sly WS, and Valle D, pp 2863-2913, McGraw-Hill, New York, 2001

2) Mabuchi H, Miyamoto S, Ueda K, Oota M, Takegoshi T, Wakasugi T, and Takeda R: Causes of death in patients with familial hypercholesterolemia. Atherosclerosis, 1986; 61:1-6

3) Hoeg JM: Homozygous familial hypercholesterolemia: a paradigm for phenotypic variation. Am J Cardiol, 1993; 72:11D-14D

4) Hokanson JE and Austin MA: Plasma triglyceride level is a risk factor for cardiovascular disease independent of high-density lipoprotein cholesterol level: a meta-analysis of population-based prospective studies. J Cardiovasc Risk, 1996; 3:213-219

5) Criqui MH, Heiss G, Cohn R, Cowan LD, Suchindran CM, Bangdiwala S, Kritchevsky S, Jacobs DR Jr., O'Grady $\mathrm{HK}$, and Davis CE: Plasma triglyceride level and mortality from coronary heart disease. N Engl J Med, 1993; 328:1220-1225

6) Campos E, Nakajima K, Tanaka A, and Havel RJ: Properties of an apolipoprotein E-enriched fraction of triglyceride-rich lipoproteins isolated from human blood plasma with a monoclonal antibody to apolipoprotein B-100. J Lipid Res, 1992; 33:369-380

7) Nakajima K, Saito T, Tamura A, Suzuki M, Nakano T, Adachi M, Tanaka A, Tada N, Nakamura H, Campos E, and Havel RJ: Cholesterol in remnant-like lipoproteins in human serum using monoclonal anti apoB-100 and anti apo A-I immunoaffinity mixed gels. Clin Chim Acta, $1993 ; 223: 53-71$

8) McNamara JR, Shah PK, Nakajima K, Cupples LA, Wilson PW, Ordovas JM, and Schaefer EJ: Remnant-like particle (RLP) cholesterol is an independent cardiovascular disease risk factor in women: results from the Framingham Heart Study. Atherosclerosis, 2001; 154:229-236

9) Masuoka H, Kamei S, Ozaki M, Kawasaki A, Shintani U, Ito $\mathrm{M}$, and Nakano T: Predictive value of remnant-like particle cholesterol as an indicator of coronary artery stenosis in patients with normal serum triglyceride levels. Intern Med, 2000; 39:540-546

10) Masuoka H, Kamei S, Wagayama H, Ozaki M, Kawasaki A, Tanaka T, Kitamura M, Katoh S, Shintani U, Misaki M, Sugawa M, Ito M, and Nakano T: Association of remnant-like particle cholesterol with coronary artery disease in patients with normal total cholesterol levels. Am Heart J, 2000; 139:305-310

11) Watts GF: Postprandial lipaemia in familial hypercholesterolaemia: clinical and metabolic significance. Atherosclerosis, 2000; 148:426-428

12) Gardner CD, Fortmann SP, and Krauss RM: Association of small low-density lipoprotein particles with the incidence of coronary artery disease in men and women. JAMA, 1996; 276:875-881

13) Lamarche B, Tchernof A, Moorjani S, Cantin B, Dagenais GR, Lupien PJ, and Després JP: Small, dense lowdensity lipoprotein particles as a predictor of the risk of ischemic heart disease in men. Prospective results from the Québec Cardiovascular Study. Circulation, 1997; 95:6975

14) Koba S, Hirano T, Yoshino G, Sakai K, Sakaue T, Adachi M, and Katagiri T: Remarkably high prevalence of small dense low-density lipoprotein in Japanese men with coronary artery disease, irrespective of the presence of diabetes. Atherosclerosis, 2002; 160:249-256

15) Krauss RM and Burke DJ: Identification of multiple subclasses of plasma low density lipoproteins in normal humans. J Lipid Res, 1982; 23:97-104

16) Hirano T, Ito $Y$, Saegusa $H$, and Yoshino G: A novel and simple method for quantification of small, dense LDL. J Lipid Res, 2003; 44:2193-2201

17) Hirano T, Ito $Y$, and Yoshino G: Measurement of small dense low-density lipoprotein particles. J Atheroscler Thromb, 2005; 12:67-72

18) Hirano T, Ito Y, Koba S, Toyoda M, Ikejiri A, Saegusa H, Yamazaki J, and Yoshino G: Clinical significance of small dense low-density lipoprotein cholesterol levels determined by the simple precipitation method. Arterioscler Thromb Vasc Biol, 2004; 24:558-563

19) Kajinami K, Koizumi J, Ueda K, Miyamoto S, Takegoshi T, and Mabuchi H: Effects of NK-104, a new hydroxymethylglutaryl-coenzyme reductase inhibitor, on lowdensity lipoprotein cholesterol in heterozygous familial hypercholesterolemia. Hokuriku NK-104 Study Group. Am J Cardiol, 2000; 85:178-183

20) Wierzbicki AS, Lumb PJ, Semra YK, and Crook MA: High-dose atorvastatin therapy in severe heterozygous familial hypercholesterolaemia. QJM, 1998; 91:291-294

21) Schneider JG, von Eynatten M, Parhofer KG, Volkmer 
JE, Schiekofer S, Hamann A, Nawroth PP, and Dugi KA: Atorvastatin improves diabetic dyslipidemia and increases lipoprotein lipase activity in vivo. Atherosclerosis, 2004; 175:325-331

22) Mabuchi H, Ito $S$, Haba $T$, Ueda $K$, and Ueda R: Discrimination of familial hypercholesterolemia and secondary hypercholesterolemia by Achilles' tendon thickness. Atherosclerosis, 1977; 28:61-68

23) Yamada S, Inoue K, Morishita R, Ogihara T, Kubono K, Kubo N, Abe A, and Sakurabayashi I: A new Lp(a) assay that is unaffected by apo(a) size polymorphism. Clin Chim Acta, 1999; 287:29-43

24) Stampfer MJ, Krauss RM, Ma J, Blanche PJ, Holl LG, Sacks FM, and Hennekens CH: A prospective study of triglyceride level, low-density lipoprotein particle diameter, and risk of myocardial infarction. JAMA, 1996; 276:882888

25) Talmud PJ, Edwards KL, Turner CM, Newman B, Palmen JM, Humphries SE, and Austin MA: Linkage of the cholesteryl ester transfer protein (CETP) gene to LDL particle size: use of a novel tetranucleotide repeat within the CETP promoter. Circulation, 2000; 101:2461-2466

26) Chung BH, Segrest JP, and Franklin F: In vitro production of beta-very low density lipoproteins and small, dense low density lipoproteins in mildly hypertriglyceridemic plasma: role of activities of lecithin: cholester acyltransferase, cholesterylester transfer proteins and lipoprotein lipase. Atherosclerosis, 1998; 141:209-225

27) Carr MC, Ayyobi AF, Murdoch SJ, Deeb SS, and Brunzell JD: Contribution of hepatic lipase, lipoprotein lipase, and cholesteryl ester transfer protein to LDL and HDL heterogeneity in healthy women. Arterioscler Thromb Vasc Biol, 2002; 22:667-673

28) Hogue JC, Lamarche B, Gaudet D, Lariviére M, Tremblay AJ, Bergeron J, Lemieux I, Després JP, Gagné C, and Couture P: Relationship between cholesteryl ester transfer protein and LDL heterogeneity in familial hypercholesterolemia. J Lipid Res, 2004; 45:1077-1083

29) Hogue JC, Lamarche B, Gaudet D, Tremblay AJ, Després JP, Gagné C, and Couture P: Genotype of the mutant LDL receptor allele is associated with LDL particle size heterogeneity in familial hypercholesterolemia. Atherosclerosis, 2006; 184:163-170

30) Nozue T, Michishita I, Ishibashi Y, Ito S, Iwaki T, Mizuguchi I, Miura M, Ito Y, and Hirano T: Small dense lowdensity lipoprotein cholesterol is a useful marker of metabolic syndrome in patients with coronary artery disease. J Atheroscler Thromb, 2007; 14:202-207

31) Tokuno A, Hirano T, Hayashi T, Mori Y, Yamamoto T, Nagashima M, Shiraishi Y, Ito Y, and Adachi M: The effects of statin and fibrate on lowering small dense LDLcholesterol in hyperlipidemic patients with type 2 diabetes. J Atheroscler Thromb, 2007; 14:128-132

32) Guerin M, Egger P, Soudant C, Le Goff W, van Tol A, Dupuis R, and Chapman MJ: Dose-dependent action of atorvastatin in type II B hyperlipidemia: preferential and progressive reduction of atherogenic apoB-containing lipoprotein subclasses (VLDL-2, IDL, small dense LDL) and stimulation of cellular cholesterol efflux. Atherosclerosis, 2002; 163:287-296
33) Lariviere $M$, Lamarche B, Pirro M, Hogue JC, Bergeron J, Gagné C, and Couture P: Effects of atorvastatin on electrophoretic characteristics of LDL particles among subjects with heterozygous familial hypercholesterolemia. Atherosclerosis, 2003; 167:97-104

34) Hoogerbrugge $\mathrm{N}$ and Jansen $\mathrm{H}$ : Atorvastatin increases low-density lipoprotein size and enhances high-density lipoprotein cholesterol concentration in male, but not in female patients with familial hypercholesterolemia. Atherosclerosis, 1999; 146:167-174

35) Yoshino G, Hirano T, and Kazumi T: Treatment of small dense LDL. J Atheroscler Thromb, 2002; 9:266-275

36) de Sauvage Nolting PR, Twickler MB, Dallinga-Thie GM, Buirma RJ, Hutten BA, and Kastelein JJ: Examination of Probands and Relatives in Statin Studies with Familial Hypercholesterolemia (ExPRESS) Study Group: Elevated remnant-like particles in heterozygous familial hypercholesterolemia and response to statin therapy. Circulation, 2002; 106:788-792

37) Twickler TB, Dallinga-Thie GM, de Valk HW, Schreuder PC, Jansen H, Cabezas MC, and Erkelens DW: High dose of simvastatin normalizes postprandial remnantlike particle response in patients with heterozygous familial hypercholesterolemia. Arterioscler Thromb Vasc Biol, 2000; 20:2422-2427

38) Stein DT, Devaraj S, Balis D, Adams-Huet B, and Jialal I: Effect of statin therapy on remnant lipoprotein cholesterol levels in patients with combined hyperlipidemia. Arterioscler Thromb Vasc Biol, 2001; 21:2026-2031

39) Horton JD, Shimano H, Hamilton RL, Brown MS, and Goldstein JL: Disruption of LDL receptor gene in transgenic SREBP-1a mice unmasks hyperlipidemia resulting from production of lipid-rich VLDL. J Clin Invest, 1999; 103:1067-1076

40) Bradley WA, Hwang SL, Karlin JB, Lin AH, Prasad SC, Gotto AM Jr., and Gianturco SH: Low-density lipoprotein receptor binding determinants switch from apolipoprotein $\mathrm{E}$ to apolipoprotein B during conversion of hypertriglyceridemic very-low-density lipoprotein to lowdensity lipoproteins. J Biol Chem, 1984; 259:14728-14735

41) de Grooth GJ, Smilde TJ, Van Wissen S, Klerkx AH, Zwinderman AH, Fruchart JC, Kastelein JJ, Stalenhoef $\mathrm{AF}$, and Kuivenhoven JA: The relationship between cholesteryl ester transfer protein levels and risk factor profile in patients with familial hypercholesterolemia. Atherosclerosis, 2004; 173:261-267

42) Suehiro T, Ohguro T, Sumiyoshi R, Yasuoka N, Nakauchi Y, Kumon Y, and Hashimoto K: Relationship of low-density lipoprotein particle size to plasma lipoproteins, obesity, and insulin resistance in Japanese men. Diabetes Care, 1995; 18:333-338

43) Kazumi T, Kawaguchi A, Hozumi T, Nagao M, Iwahashi M, Hayakawa M, Ishihara K, and Yoshino G: Low density lipoprotein particle diameter in young, nonobese, normolipidemic Japanese men. Atherosclerosis, 1999; 142:113 119

44) Iwai M, Yoshino G, Matsushita M, Morita M, Matsuba K, Kazumi T, and Baba S: Abnormal lipoprotein composition in normolipidemic diabetic patients. Diabetes Care, 1990; 13:792-796 